\title{
Gradient Bounds for Almost Complex Special Lagrangian Equation with Supercritical Phase
}

\author{
Jiaogen Zhang* \\ School of Mathematical Sciences, University of Science and Technology of China, \\ Hefei 230026, China.
}

Received September 13, 2020; Accepted December 29, 2020;

Published online January 13, 2022.

\begin{abstract}
In this article, we will consider the Dirichlet problem for special Lagrangian equation on $\Omega \subset M$, where $(M, J)$ is a compact almost complex manifold. Under the existence of $C^{2}$-smooth strictly J-plurisubharmonic subsolution $\underline{u}$, in the supercritical phase case, we obtain a uniform global gradient estimate.
\end{abstract}

AMS subject classifications: 53C07, 32Q60, 35B50.

Key words: Special Lagrangian equation, almost complex manifold, gradient estimates, maximum principle.

\section{Introduction}

In this paper, we consider the Dirichlet problem for the special Lagrangian operator, in the real setting which can be written as the the form

$$
\sum_{i} \arctan \lambda_{i}\left(D^{2} u\right)=\Theta
$$

Here $\Theta$ is a topological constant called the phase angle, $D^{2} u$ is the real Hessian of $u$. Under the coordinate system $\left\{x_{1}, \cdots, x_{n}\right\}, D^{2} u$ can be regarded as the matrix $\left\{\frac{\partial^{2} u}{\partial x_{i} \partial x_{j}}\right\}$. After an elementary orthogonal transformation,

$$
\left(\frac{\partial^{2} u}{\partial x_{i} \partial x_{j}}\right)=\operatorname{diag}\left(\lambda_{1}, \cdots, \lambda_{n}\right), \quad \lambda_{i}:=\lambda_{i}\left(D^{2} u\right)
$$

If $\Theta \in\left((n-2) \frac{\pi}{2}, n \frac{\pi}{2}\right)$ (respectively $\left.\Theta \in\left((n-1) \frac{\pi}{2}, n \frac{\pi}{2}\right)\right)$, then we called Equation (1.1) as the special Lagrangian equation with supercritical (respectively hypercritical) phase.

*Corresponding author. Email address: zjgmath@mail . ustc.edu . cn (Zhang J) 
Eq. (1.1) was introduced by Harvey-Lawson [10] when they studied the Calibrated geometry. In this case, the gradient graph $x \mapsto(x, D u(x))$ defines a calibration and also determines a Lagrangian graph, which is a minimal submanifold of $\mathbb{R}^{n} \times \mathbb{R}^{n}$. In fact, any $C^{2}$ Lagrangian submanifold $M^{2 n}$ is locally represented by a gradient graph $(x, D u(x))$ over its tangent plane. The interested reader can also refer to the Warren's PhD thesis [25] for more details of this topic. The investigation of Lagrangian manifolds also has many important applications in both geometry and physics, especially the works done by Strominger et al. [24] about mirror symmetry, which gave a very geometric picture of how mirror manifolds are connected.

In the real setting, the Dirichlet problem for Eq. (1.1) on smooth domain with strictly pseudoconvex boundary was also considered by Cafferalli-Nirenberg-Spruck [1] when $\left.\Theta \in\left((n-1) \frac{\pi}{2}, n \frac{\pi}{2}\right)\right)$. Moreover, they also showed that the special Lagrangian operator is concave in this setting. After that, the special Lagrangian equation with supercritical phase has also been studied extensively in the past few years. For instance, Warren-Yuan [26] considered the interior gradient estimates, and the interior second order estimates were obtained by Wang-Yuan [27]. For special Lagrangian equations with more general phases, one can refer a serious works of Harvey-Lawson $[11,12,14]$ et al. and references therein.

At the same time, in the complex setting, there were also many excellent works. For instance, Collins-Picard-Wu [4] obtained the existence and regularity theorems under the existence of subsolution for the Dirichlet problem, in both of real and complex cases. Dinew-Do-Tô [8] also obtained a continuous viscosity solution by using the classical Perron's envelope method. The complex special Lagrangian equation has an intimate connection with the deformed Hermitian-Yang-Mills equation. The interested reader can refer to [17-19] et al. for more profound understanding.

It is remarkable that the almost complex manifold has been studied extensively during past few years, which is motivated by differential geometry and mathematical physics $([6,13]$ and references therein). In the current note, we wish to investigate the Dirichlet problem for special Lagrangian equation on the almost complex manifold.

Let $(M, J)$ be a compact almost complex manifold of real dimension $2 n$, and $\Omega \subset M$ be a smooth domain with smooth boundary $\partial \Omega$. Fix a Hermitian metric $\omega$ on $M$. We wish to consider the Dirichlet problem for the almost complex special Lagrangian operator which can be written in the following form

$$
\begin{cases}\sum_{i} \arctan \lambda_{i}(\partial \bar{\partial} u)=h & \text { in } \Omega, \\ u=\varphi & \text { on } \partial \Omega .\end{cases}
$$

Here $\varphi, h$ are given functions on $\bar{\Omega}, \lambda_{1}, \cdots, \lambda_{n}$ are the eigenvalues of $\sqrt{-1} \partial \bar{\partial} u$ with respect to $\omega$.

We now state our main result. Assume $n \frac{\pi}{2}>h>(n-2) \frac{\pi}{2}$, i.e., the supercritical phase case, we have the following global gradient estimates, under the existence of $C^{2}$ subsolution. 
Theorem 1.1. Let $(M, J)$ be a compact almost complex manifold of real dimension $2 n$, and $\Omega \subset M$ be a smooth domain and $\varphi, h \in C^{2}(\bar{\Omega})$ with $h \in\left((n-2) \frac{\pi}{2}, n \frac{\pi}{2}\right)$. Assume $u$ is the solution for the Dirichlet problem (1.2). If there exists a strictly J-plurisubharmonic function $\underline{u} \in C^{2}(\bar{\Omega})$ such that

$$
\begin{cases}\sum_{i} \arctan \lambda_{i}(\partial \bar{\partial} \underline{u}) \geq h & \text { in } \Omega, \\ \underline{u}=\varphi & \text { on } \partial \Omega .\end{cases}
$$

Then

$$
\max _{\bar{\Omega}}\|u\|_{C^{1}(\bar{\Omega})} \leq C,
$$

where $C$ is a constant depending on $\|h\|_{C^{2}(\Omega)}, \inf _{\bar{\Omega}} h,\|\varphi\|_{C^{2}(\bar{\Omega})}$ and $\|\underline{u}\|_{C^{1}(\Omega)}$.

When we assume $\Omega \subset M$ has smooth strictly pseudoconvex boundary $\partial \Omega$ (see the terminology in the next section), we can also obtain a $C^{2}$ subsolution for (1.2) as in Lemma 2.4 below. Then we can prove the following corollary.

Corollary 1.2. Let $(M, J)$ be a compact almost complex manifold of real dimension $2 n$, and $\Omega \subset M$ be a smooth domain with smooth strictly pseudoconvex boundary $\partial \Omega$. Suppose $u$ is the solution for the Dirichlet problem (1.2) and $\varphi, h \in C^{2}(\bar{\Omega})$ with $h \in\left((n-2) \frac{\pi}{2}, n \frac{\pi}{2}\right)$. Then

$$
\max _{\bar{\Omega}}\|u\|_{C^{1}(\bar{\Omega})} \leq C,
$$

where $C$ is a constant depending on $\|h\|_{C^{2}(\Omega)}$, inf $\bar{\Omega}$ h and $\|\varphi\|_{C^{2}(\bar{\Omega})}$ and $\partial \Omega$.

Generally, the gradient estimates (1.4) or (1.5) play a crucial role in the existence theorem. About the study of fully nonlinear elliptic equations on (almost) complex manifolds, it is still a rather challenging question to derive the gradient estimates. A remarkable development was done by Dinew-Kolodziej [7]. They were able to give the gradient estimates for complex Hessian equation on Kähler manifolds by the blow up techniques and Liouville type theorems. As a consequence, they can solve it by the earlier work of Hou-Ma-Wu [15]. Very recently, Collins-Picard [3] were able to consider the Dirichlet problem for complex Hessian equations on Hermitian manifolds by making use of Dinew-Kolodziej's method. It is remarkable that Collins-Yau [5] also obtained a gradient estimate for the defomed Hermitian Yang-Mills equation in the case of base manifolds are product manifolds, by using the Phong-Sturm's trick [21].

When we further assume $n \frac{\pi}{2}>h>(n-1) \frac{\pi}{2}$, i.e., the hypercritical phase case, we can obtain the following interior second order estimates, which were proved by Huang, the author and Zhang very recently.

Theorem 1.3. ([16, Theorem 1.1]) Let $(M, J)$ be a compact almost complex manifold of real dimension $2 n$, and $\Omega \subset M$ be a smooth domain and $\varphi, h \in C^{2}(\bar{\Omega})$ with $h \in\left((n-1) \frac{\pi}{2}, n \frac{\pi}{2}\right)$. Assume $u$ is the solution for the Dirichlet problem (1.2). If there exists a strictly J-plurisubharmonic subsolution $\underline{u} \in C^{2}(\bar{\Omega})$ such that

$$
\begin{cases}\sum_{i} \arctan \lambda_{i}(\partial \bar{\partial} \underline{u}) \geq h & \text { in } \Omega, \\ \underline{u}=\varphi & \text { on } \partial \Omega .\end{cases}
$$


Then

$$
\max _{\bar{\Omega}}\|u\|_{C^{2}(\bar{\Omega})} \leq C\left(1+\max _{\partial \Omega}|\partial \bar{\partial} u|\right),
$$

where $C$ is a constant depending on $\|h\|_{C^{2}(\Omega)}, \inf _{\bar{\Omega}} h$, $\varphi$ and $\|\underline{u}\|_{C^{2}(\Omega)}$.

Remark 1.1. The boundary $C^{2}$ estimates, especially the double normal estimate, are still open for us. It might be of interest to consider the boundary $C^{2}$ estimates under the conditions in Theorem 1.3.

Furthermore, the hypercritical phase condition in Theorem 1.3 is seemed extremely important to us. We hope the arguments in [16] can be applied to derive the interior second order estimates in the supercritical phase setting.

\section{Preliminaries}

Assume $(M, J)$ is an almost complex manifold of complex dimension $2 n$, where $J$ is the almost complex structure on $M$. Let $\mathcal{A}^{p, q}$ be the set of smooth sections of $(p, q)$ form on $M$ and

$$
\mathcal{A}^{k}=\bigoplus_{p+q=k} \mathcal{A}^{p, q}
$$

We consider the exterior derivative $d: \mathcal{A}^{k} \rightarrow \mathcal{A}^{k+1}$ which satisfies $d^{2}=0$. Set $\Pi_{p+1, q}, \Pi_{p, q+1}$, $\Pi_{p+2, q-1}, \Pi_{p-1, q+2}$ be the projection $\mathcal{A}^{k+1}$ to $\mathcal{A}^{p+1, q}, \mathcal{A}^{p, q+1}, \mathcal{A}^{p+2, q-1}, \mathcal{A}^{p-1, q+2}$ respectively. Then $d$ can be divided as the following four parts:

$$
d=\partial+\bar{\partial}+T+\bar{T}
$$

Here

$$
\partial=\Pi_{p+1, q} \circ d, \quad \bar{\partial}=\Pi_{p, q+1} \circ d, \quad T=\Pi_{p+2, q-1} \circ d, \quad \bar{T}=\Pi_{p-1, q+2} \circ d .
$$

In particular, for $u \in C^{2}(M, \mathbb{R})$, then $\bar{\partial} u \in \mathcal{A}^{0,1}$ and

$$
d \bar{\partial} u=\partial \bar{\partial} u+\bar{\partial}^{2} u+T \bar{\partial} u .
$$

Taking complex conjugate and add together, then this gives

$$
T \bar{\partial} u=-\partial^{2} u, \quad \partial \bar{\partial} u=-\bar{\partial} \partial u .
$$

As a consequence, $\sqrt{-1} \partial \bar{\partial} u \in \wedge_{\mathbb{R}}^{1,1} M$.

Using the same notation as [20,22], let $e_{1}, \cdots, e_{n}$ be a local $g$-orthonormal frame of $T_{1,0} M$, for each $v \in C^{2}(M, \mathbb{R})$, we can (locally) define

$$
v_{k \bar{l}}=\sqrt{-1} \partial \bar{\partial} v\left(e_{k}, \bar{e}_{l}\right)=e_{k} \bar{e}_{l} v-\left[e_{k}, \bar{e}_{l}\right]^{0,1} v .
$$

Then we have

$$
\sqrt{-1} \partial \bar{\partial} v=\sqrt{-1} \sum v_{k l} e_{k}^{*} \wedge \bar{e}_{l}^{*}
$$


where $e_{1}^{*}, \cdots, e_{n}^{*} ; \bar{e}_{1}^{*}, \cdots, \bar{e}_{n}^{*}$ is a local $g$-orthonormal frame of $T_{\mathbb{C}}^{*} M$ dual to the base $e_{1}, \cdots, e_{n} ; \bar{e}_{1}$, $\cdots, \bar{e}_{n}$ of $T_{\mathbb{C}} M$.

Fix a Hermitian matrix $\omega$ on $M$. Define $U_{j}^{k}=\sum_{l} g^{k \bar{l}} u_{j \bar{l}}$. Based on this fact, we can rewrite Eq. in (1.2) as

$$
F(u)=f\left(\lambda_{1}, \cdots, \lambda_{n}\right)=\sum_{i} \arctan \lambda_{i}\left(U_{j}^{k}\right)=h .
$$

Let

$$
\Gamma=\left\{\lambda \in \mathbb{R}^{n}: \sum_{i} \arctan \lambda_{i}>(n-2) \frac{\pi}{2}\right\} .
$$

And for each $\sigma \in\left((n-2) \frac{\pi}{2}, n \frac{\pi}{2}\right)$, define

$$
\Gamma^{\sigma}=\left\{\lambda \in \Gamma: \sum_{i} \arctan \lambda_{i}>\sigma\right\}
$$

Then $\Gamma^{\sigma}$ is an open convex set, and $\partial \Gamma^{\sigma}=f^{-1}(\sigma)$ is a smooth convex hypersurface, see [4, Lemma 2.1]. We have following important observation.

Lemma $2.1([2,4])$. Suppose $h \in\left[(n-2) \frac{\pi}{2}+\delta, n \frac{\pi}{2}\right)$ for some $0<\delta<\frac{\pi}{2}$ and $\lambda_{1} \geq \cdots \geq \lambda_{n}$. Then there exists a constant $C_{\delta}>0$ depending only on $\delta$ such that we have the following properties

1. $\lambda_{1} \geq \cdots \geq \lambda_{n-1}>0,\left|\lambda_{n}\right| \leq \lambda_{n-1}$ and $\lambda_{n} \geq-C_{\delta}$,

2. $\sum_{i} \lambda_{i} \geq 0$,

3. $f_{i}(\lambda)=\frac{\partial f(\lambda)}{\partial \lambda_{i}}>0$ for each $i,-e^{-L f(\lambda)}$ is concave for $L \geq C_{\delta}$,

4. $\lambda_{n} \geq C_{\delta}^{-1}$ if we further assume $h \in\left[(n-1) \frac{\pi}{2}+\delta, n \frac{\pi}{2}\right)$.

We define

$$
G=-e^{-L F}, \quad \tilde{h}=-e^{-L h}
$$

And we also let

$$
F^{i \bar{j}}(u)=\frac{\partial F(u)}{\partial u_{i \bar{j}}}, \quad F^{i \bar{k}, j \bar{l}}(u)=\frac{\partial^{2} F(u)}{\partial u_{i \bar{k}} \partial u_{j \bar{l}}} .
$$

For each point $x_{0} \in \Omega$, let $\left\{e_{i}\right\}_{i=1}^{n}$ be a local unitary frame (with respect to $\omega$ ) such that $u_{i \bar{j}}\left(x_{0}\right)=\delta_{i j} u_{i \bar{i}}\left(x_{0}\right)$. We denote $u_{i \bar{i}}\left(x_{0}\right)$ by $\lambda_{i}$ for simplicity. It is useful to order $\left(\lambda_{1}, \lambda_{2}, \cdots, \lambda_{n}\right)$ such that

$$
\lambda_{1} \geq \lambda_{2} \geq \cdots \geq \lambda_{n}
$$

Then at $x_{0}$, we have

$$
F^{i \bar{j}}=F^{i \bar{i}} \delta_{i j}=\frac{1}{1+\lambda_{i}^{2}} \delta_{i j}
$$


and

$$
F^{i \bar{k}, j \bar{l}}= \begin{cases}-\frac{2 \lambda_{i}}{\left(1+\lambda_{i}^{2}\right)^{2}}, & \text { if } i=j=k=l ; \\ -\frac{\lambda_{i}+\lambda_{k}}{\left(1+\lambda_{i}^{2}\right)\left(1+\lambda_{k}^{2}\right)}, & \text { if } i=l, k=j, i \neq k ; \\ 0, & \text { otherwise. }\end{cases}
$$

We can also easily compute on $\partial \Gamma^{h}$,

$$
G^{i \bar{j}}=L e^{-L h} F^{i \bar{j}}, \quad G^{i \bar{k}, j \bar{l}}=L e^{-L h} F^{i \bar{k}, j \bar{l}}-L^{2} e^{-L h} F^{i \bar{k}} F^{j \bar{l}} .
$$

Let us define the operator

$$
\mathcal{L}=\sum_{i, j} G^{i \bar{j}}\left(e_{i} \bar{e}_{j}-\left[e_{i}, \bar{e}_{j}\right]^{0,1}\right), \quad \mathcal{G}=\sum_{i, j} G^{i \bar{j}} g_{i \bar{j}} .
$$

Definition 2.1. For $v \in C^{2}(M, \mathbb{R})$,

1. we say $v$ is $J$-plurisubharmonic on an open set $O \subset M$ if the matrix $\left\{v_{i j}\right\}$ is a nonnegative matrix at each point of $O$,

2. we say $v$ is strictly J-plurisubharmonic on an open set $O$ if for each $\varphi \in C^{2}(O)$, there exists $\varepsilon_{0}>0$ such that $u+\varepsilon \varphi$ is J-plurisubharmonic on $O$ for all $0<\varepsilon<\varepsilon_{0}$.

For other upper semi-continuous functions, we can also define the terminology of $J$-plurisubharmonic as in [13]. We denote the set of $J$-plurisubharmonic functions by $\operatorname{PSH}(\Omega)$.

Since $\underline{u}$ is strictly J-plurisubharmonic, then there exists a uniform constant $\tau>0$ such that

$$
\tau^{-1} \omega \geq \sqrt{-1} \partial \bar{\partial} \underline{u} \geq \tau \omega
$$

\subsection{Basic technique}

The following lemma is due to Collins-Picard-Wu [4, Lemma 3.1 and Corollary 3.2], which is inspired from Székelyhidi [23]. One can also refer Guan [9, Theorem 2.16] for more general fully nonlinear elliptic PDEs.

Lemma 2.2. Suppose that $h \in\left((n-2) \frac{\pi}{2}, \frac{n \pi}{2}\right)$. Let $\underline{u} \in C^{2}(\Omega)$ be a strictly J-plurisubharmonic function which is also the subsolution to (1.2). There exist positive constants $N, \theta>0$ such that if $\sum_{i} u_{i \bar{i}} \geq N$ at a point $p \in \Omega$ such that $g_{i \bar{j}}=\delta_{i j}$ and the matrix $\left\{u_{i j}\right\}$ is diagonal, then we have

$$
\mathcal{L}(\underline{u}-u) \geq \theta\left(\sum_{i=1}^{n} G^{i \bar{i}}+1\right)
$$

and

$$
\sum_{i=1}^{n} G^{i \bar{i}} \geq \theta
$$




\subsection{Comparison principle}

Lemma 2.3. Let $\Omega \subset M$ be a bounded domain. If $u, v \in C^{2}(\bar{\Omega}) \cap P S H(\Omega)$ such that $u$ is strictly J-plurisubharmonic and

$$
G(u) \geq G(v) .
$$

Then $u-v$ attains its maximum on $\partial \Omega$.

Proof. By direct calculating,

$$
\begin{aligned}
0 & \leq G(u)-G(v) \\
& =\int_{0}^{1} \frac{d}{d t} G(t u+(1-t) v) d t \\
& =\sum \int_{0}^{1} \tilde{G}^{i \bar{j}}(t) d t\left(u_{i j}-v_{i j}\right) \\
& =L_{0}(u-v),
\end{aligned}
$$

for some second order operator $L_{0}$, where $\tilde{G}^{i \bar{j}}(t)$ are cofactor of $G_{i \bar{j}}(t u+(1-t) v)$. Note that the matrix $\left\{\int_{0}^{1} \tilde{G}^{i \bar{j}}(t) d t\right\}$ is positive definite since $u$ is strictly $J$-plurisubharmonic. Hence, $L_{0}$ is elliptic. With the aid of standard elliptic PDE theory, $u-v$ attains its maximum on $\partial \Omega$.

\subsection{Existence of subsolution}

Definition 2.2. We say $\partial \Omega$ is strictly pseudonconvex if there is a smooth defining function $\rho$, i.e., $\rho<0$ in $\Omega, \rho=0, \nabla \rho \neq 0$ on $\partial \Omega$ and $\rho$ is strictly J-plurisubharmonic.

It is remarkable that Harvey and Lawson [13] had proved that on any point of a complex manifolds, there exist a neighborhood systems with strictly pseudoconvex smooth boundaries.

In this case, there exists a constant $m(\rho)>0$ defined as the smallest constant $m$ such that $m \sqrt{-1} \partial \bar{\partial} \rho>\omega$, i.e.,

$$
m(\rho)=\min \{m>0: m \sqrt{-1} \partial \bar{\partial} \rho>\omega\} .
$$

Let $\tilde{\varphi} \in C^{2}(\bar{\Omega})$ solve the following Dirichlet problem:

$$
\left\{\begin{array}{l}
\mathcal{L}(\tilde{\varphi})=0 \text { in } \Omega, \\
\tilde{\varphi}=\varphi \text { on } \partial \Omega .
\end{array}\right.
$$

That is, $\tilde{\varphi}$ can be regarded as the $\mathcal{L}$-harmonic extension of $\varphi_{\mid \partial \Omega}$.

We have the following lemma: 
Lemma 2.4. There exists a constant $R(\rho)$ depending on $m(\rho)$ such that

$$
u_{R}:=\tilde{\varphi}+R \rho
$$

satisfies

$$
\sum_{i} \arctan \lambda_{i}\left(\partial \bar{\partial} u_{R}\right)>h \text { for all } R \geq R(\rho) .
$$

Therefore, $u_{R}$ in (2.6) is our desired subsolution for Eq. (1.2) since $u_{R}=\varphi$ on $\partial \Omega$.

\section{$3 C^{0}$ and $C^{1}$ estimates}

\subsection{Uniform estimates}

Lemma 3.1. Let $u$ (respectively $\underline{u}$ ) be the solution (respectively subsolution) for (1.2). We have

$$
\underline{u} \leq u \leq \tilde{\varphi} .
$$

Therefore, there exists a constat $C$ depending on $\|\underline{u}\|_{L^{\infty}(\Omega)},\|h\|_{L^{\infty}(\Omega)},\|\varphi\|_{L^{\infty}(\Omega)}$, such that

$$
\|u\|_{L^{\infty}(\Omega)} \leq C
$$

Proof. On the one hand, since $\underline{u}$ is a subsolution for (1.2), the first inequality is easily from Lemma 2.3. On the other hand, we can show that $u$ is a subsolution for (2.5) since $\mathcal{L}(u)=h$, then by the classical maximum principle we also have the second inequality.

\subsection{Boundary gradient estimates}

Lemma 3.2. Let $u$ (respectively $\underline{u}$ ) be the solution (respectively subsolution) for (1.2). We have

$$
|u|_{C^{0,1}(\partial \Omega)} \leq C
$$

where $C$ is a constant depending on the $\|\underline{u}\|_{C^{1}(\bar{\Omega})}$ and $\varphi$.

Proof. From the previous lemma, $u, \underline{u}$ and $\tilde{\varphi}$ have the same boundary value $\varphi_{\mid \partial \Omega}$, then it is easy to verify $|\nabla u|(z) \leq \sup \{|\nabla \underline{u}|(z),|\nabla \tilde{\varphi}|(z)\}$ for each $z \in \partial \Omega$.

\subsection{Interior gradient estimates}

Proposition 3.1. Let $u$ (respectively $\underline{u}$ ) be the solution (respectively subsolution) for (1.2). Then

$$
|u|_{C^{0,1}(\Omega)} \leq C .
$$

for some positive constant $C$ depending on the allowed data and $\|\underline{u}\|_{C^{1}(\bar{\Omega})},\|u\|_{C^{0}(\bar{\Omega})},\|u\|_{C^{0,1}(\partial \Omega)}$. 
Proof. Let

$$
H(\eta)=A e^{B \eta}, \quad \eta=\underline{u}-u .
$$

Here $A, B>0$ are certain constants to be picked up later. In this proof, the $C$ below denotes the constants those may change from line to line, and it depends on $A, B$ that we yet to choose. Consider the test function

$$
V=e^{H}|\nabla u|^{2}
$$

Suppose $V$ achieves maximum at the $x_{0}$ in the interior of $\Omega$, otherwise we are done. Then at $x_{0}$, we can choose a proper local frame still denoted by $e_{1}, \cdots, e_{n}$ such that $g_{i j}=\delta_{i j}$ and the matrix $\left\{u_{i j}\right\}$ is diagonal.

Using the maximum principle, it follows that

$$
\begin{aligned}
0 \geq & \frac{\mathcal{L}(V)\left(x_{0}\right)}{B H e^{H}|\nabla u|^{2}} \\
= & \frac{\mathcal{L}\left(e^{H}\right)}{B H e^{H}}+\frac{\mathcal{L}\left(|\nabla u|^{2}\right)}{B H|\nabla u|^{2}}+2 \sum_{i} G^{i \bar{i}} \operatorname{Re}\left\{e_{i}(H) \frac{\bar{e}_{i}\left(|\nabla u|^{2}\right)}{B H|\nabla u|^{2}}\right\} \\
= & \mathcal{L}(\eta)+B(1+H) \sum_{i} G^{i \bar{i}}\left|e_{i}(\eta)\right|^{2}+\frac{\mathcal{L}\left(|\nabla u|^{2}\right)}{B H|\nabla u|^{2}} \\
& +\frac{2}{|\nabla u|^{2}} \sum_{i, j} G^{i \bar{i}} \operatorname{Re}\left\{e_{i}(\eta) \bar{e}_{i} e_{j}(u) \bar{e}_{j}(u)+e_{i}(\eta) \bar{e}_{i} \bar{e}_{j}(u) e_{j}(u)\right\} .
\end{aligned}
$$

By direct calculations,

$$
\mathcal{L}\left(|\nabla u|^{2}\right)=\sum_{i} G^{i \bar{i}}\left(e_{i} e_{\bar{i}}\left(|\nabla u|^{2}\right)-\left[e_{i}, \bar{e}_{i}\right]^{0,1}\left(|\nabla u|^{2}\right)\right)=I+I I+I I I,
$$

where

$$
\begin{aligned}
& I=\sum_{i, j} G^{i \bar{i}}\left(e_{i} \bar{e}_{i} e_{j} u-\left[e_{i}, \bar{e}_{i}\right]^{0,1} e_{j} u\right) \bar{e}_{j} u ; \\
& I I=\sum_{i, j} G^{i \bar{i}}\left(e_{i} \bar{e}_{i} \bar{e}_{j} u-\left[e_{i}, \bar{e}_{i}\right]^{0,1} \bar{e}_{j} u\right) e_{j} u ; \\
& I I I=\sum_{i, j} G^{i \bar{i}}\left(\left|e_{i} e_{j} u\right|^{2}+\left|e_{i} \bar{e}_{j} u\right|^{2}\right) .
\end{aligned}
$$

Differentiating Eq. (2.1) with respect to each $e_{j}$, then

$$
\sum_{i} G^{i \bar{i}}\left(e_{j} e_{i} \bar{e}_{i} u-e_{j}\left[e_{i}, \bar{e}_{i}\right]^{0,1} u\right)=\tilde{h}_{j}
$$


Recall the definition of Lie bracket $e_{i} e_{j}-e_{j} e_{i}=\left[e_{i}, e_{j}\right]$, we can calculate

$$
\begin{aligned}
I+I I= & 2 \sum_{i, j} G^{i \bar{i}} \operatorname{Re}\left\{\left(e_{i} \bar{e}_{i} e_{j} u-\left[e_{i}, \bar{e}_{i}\right]^{0,1} e_{j} u\right) \bar{e}_{j}(u)\right\} \\
= & 2 \sum_{i, j} G^{i \bar{i}} \operatorname{Re}\left\{\left(e_{j} e_{i} \bar{e}_{i} u+e_{i}\left[\bar{e}_{i}, e_{j}\right] u+\left[e_{i}, e_{j}\right] \bar{e}_{i} u-\left[e_{i}, \bar{e}_{i}\right]^{0,1} e_{j} u\right) \bar{e}_{j}(u)\right\} \\
\stackrel{(3.7)}{=} & 2 \sum_{j} \operatorname{Re}\left\{e_{j}(\tilde{h}) \bar{e}_{j}(u)\right\}+2 \sum_{i, j} G^{i \bar{i}} \operatorname{Re}\left\{e_{j}\left[e_{i}, \bar{e}_{i}\right]^{0,1} u \bar{e}_{j}(u)\right\} \\
& +2 \sum_{i, j} G^{i \bar{i}} \operatorname{Re}\left\{\left(e_{i}\left[\bar{e}_{i}, e_{j}\right] u+\left[e_{i}, e_{j}\right] \bar{e}_{i} u-\left[e_{i}, \bar{e}_{i}\right]^{0,1} e_{j} u\right) \bar{e}_{j}(u)\right\} \\
= & 2 \sum_{j} \operatorname{Re}\left\{e_{j}(\tilde{h}) \bar{e}_{j}(u)\right\}+2 \sum_{i, j} G^{i \bar{i}} \operatorname{Re}\left\{\left[e_{j},\left[e_{i}, \bar{e}_{i}\right]^{0,1}\right](u) \bar{e}_{j}(u)\right\} \\
& +2 \sum_{i, j} G^{i \bar{i}} \operatorname{Re}\left\{\left(e_{i}\left[\bar{e}_{i}, e_{j}\right] u+\left[e_{i}, e_{j}\right] \bar{e}_{i} u\right) \bar{e}_{j}(u)\right\} .
\end{aligned}
$$

We may and do assume $|\nabla u| \gg 1$. Therefore, C-S denotes the Cauchy-Schwarz inequality from now on.

$$
\begin{aligned}
I+I I & \geq 2 \sum_{j} \operatorname{Re}\left\{\tilde{h}_{j} u_{j}\right\}-C|\nabla u| \sum_{i, j} G^{i \bar{i}}\left(\left|e_{i} e_{j} u\right|+\left|e_{i} \bar{e}_{j} u\right|\right)-C|\nabla u|^{2} \mathcal{G} \\
& \geq 2 \sum_{j} \operatorname{Re}\left\{\tilde{h}_{j} u_{j}\right\}-\frac{C}{\varepsilon}|\nabla u|^{2} \mathcal{G}-\varepsilon \sum_{i, j} G^{i \bar{i}}\left(\left|e_{i} e_{j} u\right|^{2}+\left|e_{i} \bar{e}_{j} u\right|^{2}\right),
\end{aligned}
$$

for each $0<\varepsilon \leq 1 / 2$, where is the last step we have used the Cauchy-Schwarz inequality. Plug (3.8) into (3.6), hence

$$
\frac{\mathcal{L}\left(|\nabla u|^{2}\right)}{B H|\nabla u|^{2}} \geq \frac{-C}{B H|\nabla u|}+(1-\varepsilon) \sum_{i, j} G^{i \bar{i}} \frac{\left|e_{i} e_{j} u\right|^{2}+\left|e_{i} \bar{e}_{j} u\right|^{2}}{B H|\nabla u|^{2}}-\frac{C \mathcal{G}}{B H \varepsilon} .
$$

Now we estimate the last term of (3.5). For $0<\varepsilon \leq \frac{1}{2}$, then $1 \leq(1-\varepsilon)(1+2 \varepsilon)$. Using the definition of Lie bracket again, we achieve

$$
\begin{array}{rl} 
& 2 \sum_{i, j} G^{i \bar{i}} \operatorname{Re}\left\{e_{i}(\eta) \bar{e}_{i} e_{j}(u) \bar{e}_{j}(u)\right\} \\
= & 2 \sum_{i, j} G^{i \bar{i}} \operatorname{Re}\left\{e_{i}(\eta) \bar{e}_{j}(u)\left\{e_{j} \bar{e}_{i}(u)-\left[e_{j}, \bar{e}_{i}\right]^{0,1}(u)-\left[e_{j}, \bar{e}_{i}\right]^{1,0}(u)\right\}\right\} \\
= & 2 \sum_{i} G^{i \bar{i}} \lambda_{i} \operatorname{Re}\left\{e_{i}(\eta) \bar{e}_{i}(u)\right\}-2 \sum_{i, j} G^{i \bar{i}} \operatorname{Re}\left\{e_{i}(\eta) \bar{e}_{j}(u)\left[e_{j}, \bar{e}_{i}\right]^{1,0}(u)\right\} \\
\mathrm{C}-S & 2 \sum_{i} G^{i \bar{i}} \lambda_{i} \operatorname{Re}\left\{e_{i}(\eta) \bar{e}_{i}(u)\right\}-\varepsilon B H|\nabla u|^{2} \sum_{i} G^{i \bar{i}}\left|e_{i}(\eta)\right|^{2}-\frac{C}{B H \varepsilon}|\nabla u|^{2} \mathcal{G}
\end{array}
$$


and

$$
\begin{aligned}
& 2 \sum_{i, j} G^{i \bar{i}} \operatorname{Re}\left\{e_{i}(\eta) \bar{e}_{i} \bar{e}_{j}(u) e_{j}(u)\right\} C-S \\
\geq & -\frac{(1-\varepsilon)}{B H} \sum_{i, j} G^{i \bar{i}}\left|\bar{e}_{i} \bar{e}_{j}(u)\right|^{2}-(1+2 \varepsilon) B H|\nabla u|^{2} \sum_{i} G^{i \bar{i}}\left|e_{i}(\eta)\right|^{2} .
\end{aligned}
$$

It follows (3.10)-(3.11) that

$$
\begin{aligned}
& 2 \sum_{i} G^{i \bar{i}} \operatorname{Re}\left\{e_{i}(H) \frac{\bar{e}_{i}\left(|\nabla u|^{2}\right)}{B H|\nabla u|^{2}}\right\} \\
& \geq \frac{2}{|\nabla u|^{2}} \sum_{i} G^{i \bar{i}} \lambda_{i} \operatorname{Re}\left\{e_{i}(\eta) \bar{e}_{i}(u)\right\}-\frac{C \mathcal{G}}{B H \varepsilon} \\
& \quad-(1+3 \varepsilon) B H \sum_{i} G^{i \bar{i}}\left|e_{i}(\eta)\right|^{2}-(1-\varepsilon) \sum_{i, j} G^{i \bar{i}} \frac{\left|\bar{e}_{i} \bar{e}_{j}(u)\right|^{2}}{B H|\nabla u|^{2}} .
\end{aligned}
$$

Coupled with the inequalities (3.5), (3.9) and (3.12), we obtain

$$
\begin{gathered}
0 \geq \mathcal{L}(\eta)+B(1-3 \varepsilon H) \sum_{i} G^{i \bar{i}}\left|e_{i}(\eta)\right|^{2}-\frac{C \mathcal{G}}{B H \varepsilon}-\frac{C}{B H|\nabla u|} \\
+\frac{2}{|\nabla u|^{2}} \sum_{i} G^{i \bar{i}} \lambda_{i} \operatorname{Re}\left\{e_{i}(\eta) \bar{e}_{i}(u)\right\} .
\end{gathered}
$$

Hence, we can choose $\varepsilon=\frac{1}{6 H\left(x_{0}\right)}$. Note that $\varepsilon \leq \frac{1}{2}$ if we let $A \geq \frac{1}{3} e^{B \sup _{\bar{\Omega}}(u-\underline{u})}$ be large enough. Then

$$
\begin{aligned}
& \mathcal{L}(\eta)+\frac{2}{|\nabla u|^{2}} \sum_{i} G^{i \bar{i}} \lambda_{i} \operatorname{Re}\left\{e_{i}(\eta) \bar{e}_{i}(u)\right\}+\frac{B}{2} \sum_{i} G^{i \bar{i}}\left|e_{i}(\eta)\right|^{2} \\
\leq & \frac{C}{B H|\nabla u|}+\frac{C \mathcal{G}}{B} .
\end{aligned}
$$

Case 1. If $\sum_{i} u_{i \bar{i}} \geq N$ for some positive constant $N$ large as in Lemma 2.2. Using the assumption $|\nabla u| \geq \max \{1,|\nabla \underline{u}|\}$ we obtain

$$
\begin{aligned}
\frac{2}{|\nabla u|^{2}} \sum_{i} G^{i \bar{i}} \lambda_{i} \operatorname{Re}\left\{e_{i}(u) \bar{e}_{i}(\eta)\right\} & \stackrel{C-S}{\geq}-\frac{B}{4} \sum_{i} G^{i \bar{i}}\left|e_{i}(\eta)\right|^{2}-\frac{C}{B|\nabla u|^{2}} \sum_{i} \frac{\lambda_{i}^{2}}{1+\lambda_{i}^{2}} \\
& \geq-\frac{B}{4} \sum_{i} G^{i \bar{i}}\left|e_{i}(\eta)\right|^{2}-\frac{C}{B|\nabla u|^{2}} .
\end{aligned}
$$

It follows (3.13) that

$$
\theta+\theta \mathcal{G} \leq \frac{C}{B H|\nabla u|}+\frac{C \mathcal{G}}{B}+\frac{C}{B|\nabla u|^{2}}
$$


Note that the terms involving $\mathcal{G}$ can be discarded for the choice of $B$ as before. Then we have

$$
\theta \leq \frac{C}{B H|\nabla u|}+\frac{C}{B|\nabla u|^{2}}
$$

This proves $|\nabla u| \leq C$.

Case 2: If $\sum_{i} u_{i \bar{i}} \leq N$. By Lemma 2.1 we can show that $\left|u_{j j}\right| \leq 2 N$ for each $j$, if $N$ is very large. Indeed, $\left|u_{j \bar{j}}\right| \leq \sum_{i} u_{i \bar{i}}+\left|u_{n \bar{n}}\right| \leq N+C_{\delta} \leq 2 N$ if we let $N \geq C_{\delta}$, since only $u_{n \bar{n}}$ might negative and others are positive. Then we have $G^{j \bar{j}} \geq \frac{1}{C\left(1+4 N^{2}\right)}$ for each $j$. Therefore,

$$
\sum_{i} G^{i \bar{i}}\left|e_{i}(\eta)\right|^{2} \geq \frac{1}{C\left(1+4 N^{2}\right)}|\nabla \eta|^{2}
$$

Plugging into (3.13) it follows

$$
\left(\tau-\frac{C}{B}\right) \mathcal{G}+\frac{B}{C\left(1+4 N^{2}\right)}|\nabla \eta|^{2} \leq \frac{C}{B H|\nabla \eta|}+C,
$$

since $|\nabla u| \geq \max \{1,|\nabla \underline{u}|\}$. By the choice of $B$ as before we have

$$
\frac{B|\nabla \eta|^{2}}{C\left(1+4 N^{2}\right)} \leq \frac{C}{B H|\nabla \eta|}+C
$$

As a consequence, $|\nabla \eta| \leq C$ and thus we deduce $|\nabla u| \leq|\nabla \eta|+|\nabla \underline{u}| \leq C$. Then the proof is complete.

\section{Acknowledgments}

The author would like to thank his advisor professor Xi Zhang for his constant encouragements. He would also like to thank the referees for many useful suggestions and comments. The author was partially supported by National Natural Science Foundation of China (Grants Nos. 11625106, 11571332 and 11721101). The research was partially supported by the project "Analysis and Geometry on Bundle" of Ministry of Science and Technology of the People's Republic of China.

\section{References}

[1] Caffarelli L, Nirenberg L, Spruck J. The Dirichlet problem for nonlinear second order elliptic equations, III: functions of the eigenvalues of the Hessian. Acta Math., 1985, 155: 261-301.

[2] Chen C, Ma X, Wei W. The Newmann problem of complex special Lagrangian equations with supercritical phase. Anal. Theory Appl., 2019, 35(2): 144-162.

[3] Collins T C, Picard S. The Dirichlet problem for the $k$-Hessian equation on a complex manifold. ArXiv:1909.00447. 
[4] Collins T C, Picard S, Wu X. Concavity of the Lagrangian phase operator and applications. Calc. Var., 2017, 56(4) 89.

[5] Collins T C, Yau S T. Moment maps, nonlinear PDE, and stability in mirror symmetry, preprint. ArXiv:1811.04824.

[6] Chu J, Tosatti $V$, Weinkove B. The Monge-Ampère equation for non-integrable almost complex structures. J. Eur. Math. Soc., 2019, 21(7): 1949-1984.

[7] Dinew S, Kołodziej S. Liouville and Calabi-Yau type theorems for complex Hessian equations. Amer. J. Math., 2017, 137(2): 403-415.

[8] Dinew S, Do H, Tô T D. A viscosity approach to the Dirichlet problem for degenerate complex Hessian type equations. Anal. PDE, 2019, 12(2): 505-535.

[9] Guan B. Second-order estimates and regularity for fully nonlinear elliptic equations on Riemannian manifolds. Duke Math. J., 2014, 163(8): 1491-1524.

[10] Harvey F R, Lawson H B J. Calibrated geometries. Acta Math., 1982, 148(1): 47-157.

[11] Harvey F R, Lawson H B J. Dirichlet duality and the non-linear Dirichlet problem. Comm. Pure Appl. Math., 2010, 62(3): 396-443.

[12] Harvey F R, Lawson H B J. Dirichlet duality and the non-linear Dirichlet problem on Riemannian manifolds. J. Diff. Geom., 2011, 88(3): 395-482.

[13] Harvey F R, Lawson H B J. Potential theory on almost complex manifolds. Ann. Inst. Fourier, 2015, 65(1): 171-210.

[14] Harvey F R, Lawson H B J. Pseudoconvexity for the special Lagrangian potential equation. ArXiv:2001.09818v1.

[15] Hou Z, Ma X, Wu D. A second order estimate for complex Hessian equations on a compact Kähler manifold. Math. Res. Lett., 2010, 17(3): 547-561.

[16] Huang L, Zhang L, Zhang X. The deformed Hermitian-Yang-Mills equation on almost Hermitian manifolds. ArXiv:2011.14091v1, 2020. To appear Sci China Math.

[17] Jacob A, Yau S T. A special Lagrangian type equation for holomorphic line bundles. Math. Ann., 2017, 369(1-2): 869-898.

[18] Leung N C, Yau S T, Zaslow E. From special Lagrangian to Hermitian-Yang-Mills via Fourier-Mukai transform. Adv. Theor. Math. Phys., 2000, 4(6): 1319-1341.

[19] Mariõ M, Minasian R, Moore G, et al. Nonlinear instantons from supersymmetric pbranes. J. High Energy Phys., 2000, 1(5): 32pp.

[20] Pali N. Fonctions plurisousharmoniques et courants positifs de type $(1,1)$ sur une variété presque complexe. Manuscripta Math., 2005, 118(3): 311-337.

[21] Phong P H, Sturm J. The Dirichlet problem for degenerate complex Monge-Ampère eqautions. Comm. Anal. Geom., 2010, 18(1): 145-170.

[22] Plis S. The Monge-Ampère equation on almost complex manifolds. Math Z., 2014, 276: 969983.

[23] Székelyhidi G. Fully nonlinear elliptic equations on compact Hermitian manifolds. J. Differ. Geom., 2018, 109(2): 337-378.

[24] Stromginer A, Yau S T, Zaslow E. Mirror symmetry is T-duality. Nuclear Phys. B., 1996, 479: 243-259.

[25] Warren M. Special Lagrangian Equation[D]. Ph.D. Thesis, University of Washington, 2008.

[26] Warren M, Yuan Y. Hessian and gradient estimates for three dimensional special Lagrangian equations with large phase. Amer. J. Math., 2010, 132(3): 751-770.

[27] Wang D, Yuan Y. Hessian estimates for special Lagrangian equations with critical and supercritical phases in general dimensions. Amer. J. Math., 2014, 136: 481-499. 